\title{
A new period determination for the close PG1159 binary SDSS J212531.92-010745.9
}

\author{
S. Schuh ${ }^{1, \star}$, I. Traulsen ${ }^{1}$, T. Nagel ${ }^{2}$, E. Reiff ${ }^{2}$, D. Homeier ${ }^{1}$, H. Schwager ${ }^{2}$, D.-J. Kusterer ${ }^{2}$, R. Lutz ${ }^{1}$, \\ and M. R. Schreiber ${ }^{3}$ \\ 1 Institut für Astrophysik, Georg-August-Universität Göttingen, Friedrich-Hund-Platz 1, 37077 Göttingen, Germany \\ 2 Institut für Astronomie und Astrophysik, Eberhard-Karls-Universität Tübingen, Sand 1, 72076 Tübingen, Germany \\ 3 Departamento de Fisica y Meteorologia, Facultad de Ciencias, Universidad de Valparaiso, Valparaiso, Chile
}

Received 30 Aug 2007, accepted later

Published online later

Key words Ephemerides - stars: AGB and post-AGB - white dwarfs - binaries: close

Methods to measure masses of PG 1159 stars in order to test evolutionary scenarios are currently based on spectroscopic masses or asteroseismological mass determinations. One recently discovered PG 1159 star in a close binary system may finally allow the first dynamical mass determination, which has so far been analysed on the basis of one SDSS spectrum and photometric monitoring.

In order to be able to phase future radial velocity measurements of the system SDSS J212531.92-010745.9, we follow up on the photometric monitoring of this system to provide a solid observational basis for an improved orbital ephemeris determination.

New white-light time series of the brightness variation of SDSS J212531.92-010745.9 with the Tübingen $80 \mathrm{~cm}$ and Göttingen $50 \mathrm{~cm}$ telescopes extend the monitoring into a second season (2006), tripling the length of overall observational data available, and significantly increasing the time base covered.

We give the ephemeris for the orbital motion of the system, based on a sine fit which now results in a period of 6.95573(5) h, and discuss the associated new amplitude determination in the context of the phased light curve variation profile. The accuracy of the ephemeris has been improved by more than one order of magnitude compared to that previously published for 2005 alone.

(c) 2007 WILEY-VCH Verlag GmbH \& Co. KGaA, Weinheim

\section{Introduction}

PG 1159 stars are hot hydrogen-deficient pre-WDs which are believed to be the outcome of a late or very late heliumshell flash during their post-AGB evolution. About 40 such objects are known at present (Werner \& Herwig, 2006), and a subset of currently 11 objects forms the class of the pulsating GW Vir variables. From their evolutionary history, typical masses should be around $0.6 \mathrm{M}_{\odot}$; spectroscopic and asteroseismological mass determinations confirm this. These methods however rely on stellar structure and evolution modelling, and can so far not be tested independently.

Nagel et al. (2006) have recently announced the unique discovery of a PG 1159 star in a close binary system, which might for the first time allow a dynamical mass determination for a PG 1159 star. SDSS J212531.92-010745.9 was discovered to show $\mathrm{H} \alpha$ emission during a systematic search of SDSS archival data for white dwarf + main sequence companion candidates. It was subsequently classified as a PG 1159 star from a spectral analysis of the SDSS spectrum, and found to undergo brightness variations in followup time-resolved photometry. The light curve shows a periodicity of $6.95616(33) \mathrm{h}$, attributable to orbital motion, with

\footnotetext{
^ Corresponding author: e-mail: schuh@ astro.physik.uni-goettingen.de
}

a flat bottom part, and no eclipses. The periodic brightening with a peak-to-peak amplitude of 0.7 mag can be interpreted as the light contribution by the irradiated side of the cool companion.

We have continued the photometric coverage of this system during the following 2006 observing season, and report the improved ephemeris with a more accurate period determination in this paper.

\section{Observations}

Following up the light curve of SDSS J212531.92-010745.9 presented by Nagel et al. (2006), we have obtained new observations with the Tübingen $80 \mathrm{~cm}$ and the Göttingen $50 \mathrm{~cm}$ telescopes during 19 nights between September and November 2006. The full data set now available is listed in Table 1 with details of the set-up.

Data reduction was done using the TRIPP routines (Schuh et al., 2003). The images were bias, dark, and flat field corrected, and relative light curves were obtained from aperture photometry using the same set of reference stars for all data sets. The flux of the combined data was normalised to its mean value, and all times were heliocentrically corrected. 


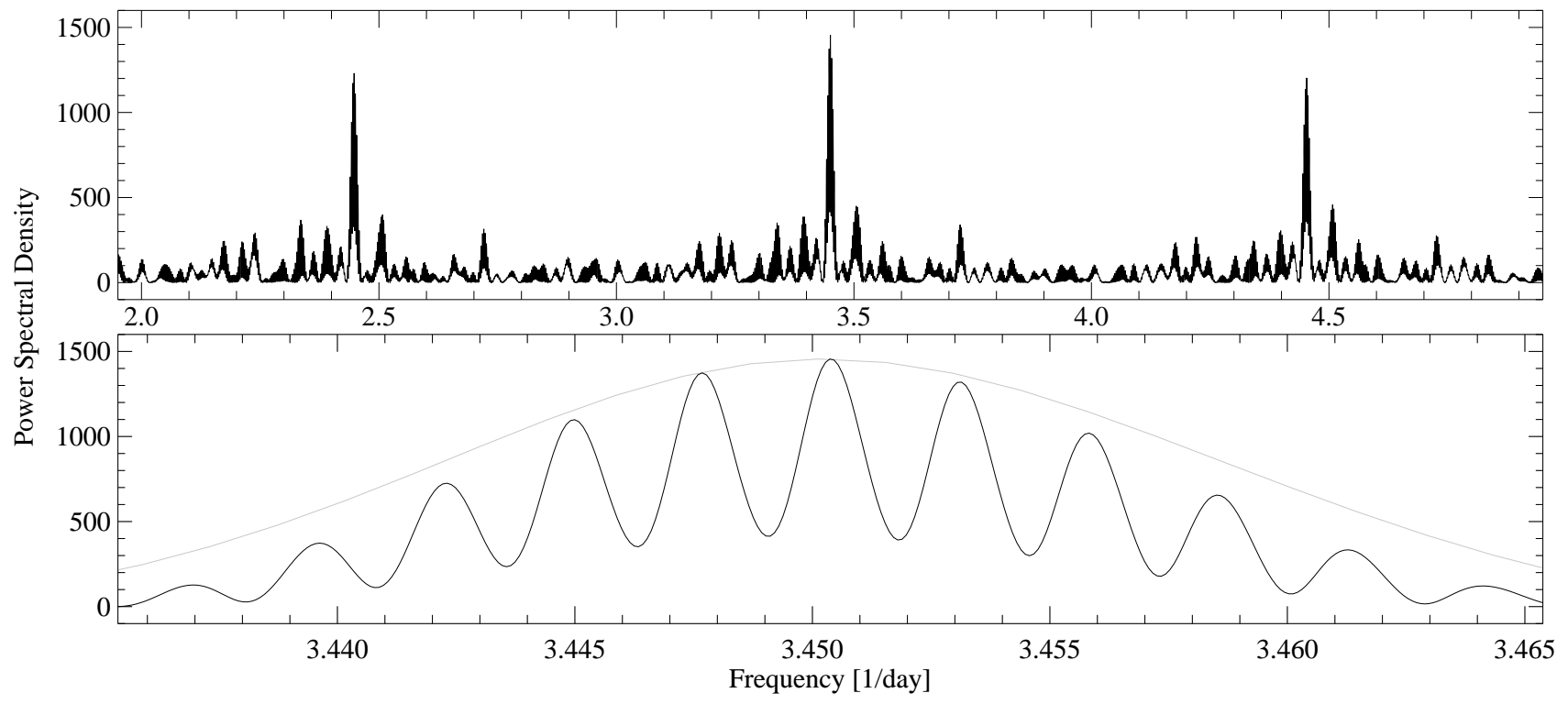

Fig. 1 Periodogram of the combined light curve: frequency range illustrating the one-day (upper panel) and the yearly alias patterns (lower panel), compared to the central peak of the periodogram obtained from the 2005 data alone (grey line).

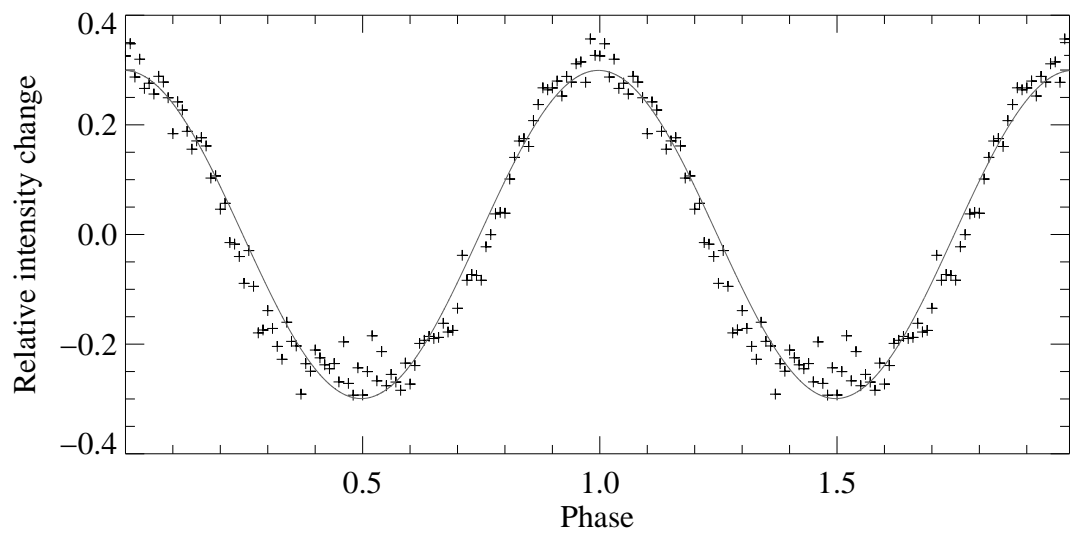

Fig. 2 Profile of the combined light curve obtained by folding it onto the orbital period and rebinning into 100 phase bins (crosses), and overplotted sine fit (grey line).

\section{Results}

In Fig.11 we show the periodogram of the combined light curve from 2005 and 2006. The correct position of the dominant frequency can unambiguously be identified from the strong alias pattern. The gain in accuracy over the 2005 data alone is illustrated by the central peak (grey line in the lower panel). By assuming the corresponding period as a start value, the combined light curve was fitted with a nonlinear least-squares sine fit. It resulted in an improved period determination of 6.95573(5) $\mathrm{h}$ and a sinusoidal amplitude of 0.299 (33) relative intensity change (0.284(28) mag). The amplitude (and its uncertainty, determined as the $1 \sigma$ residual scatter of the original data after subtraction of the mean profile) should be considered as an estimate of the magnitude of the brightness change only (for details see the Discussion section).
Referring to the most recent observation to define the zero point, we determine the ephemeris of predicted $\max$ ima times to be

$$
\mathrm{HJD}=2454055.2134(4)+0.289822(2) \cdot E .
$$

The uncertainty in the period corresponds to the $1 \sigma$ formal fitting error, while the uncertainty in the zero point corresponds to half the cycle time in the short-exposure runs.

\section{Discussion}

In Fig.2, we compare the folded profile to the sinusoidal amplitude of 0.299(33) relative intensity change. Clearly, the observed peak-to-peak variation is not fully reproduced by the fit, owing to the broader trough and the narrow maximum already described by Nagel et al. (2006), who fitted magnitude changes, while we now fit intensity changes. For a higher fit amplitude and/or raised zero line, the observed 
Table 1 Observation log. All observations were performed with clear filter.

\begin{tabular}{|c|c|c|c|c|c|}
\hline Date & $\mathrm{t}_{\exp }[s]$ & $\mathrm{t}_{\text {cycle }}[s]$ & Duration $[s]$ & Telescope & Camera \\
\hline $2005 / 09 / 21$ & 90 & 98 & 18900 & $80 \mathrm{~cm}$ & ST-7E \\
\hline $2005 / 09 / 22$ & 90 & 98 & 18899 & $80 \mathrm{~cm}$ & ST-7E \\
\hline $2005 / 09 / 23$ & 90 & 98 & 21758 & $80 \mathrm{~cm}$ & ST-7E \\
\hline $2005 / 09 / 23$ & 180 & 194 & 4051 & $50 \mathrm{~cm}$ & STL-6303E \\
\hline $2005 / 09 / 23$ & 240 & 254 & 9656 & $50 \mathrm{~cm}$ & STL-6303E \\
\hline 2005/10/06 & 240 & 248 & 10202 & $50 \mathrm{~cm}$ & STL-6303E \\
\hline 2005/10/07 & 240 & 246 & 14897 & $50 \mathrm{~cm}$ & STL-6303E \\
\hline 2005/10/08 & 240 & 248 & 9298 & $50 \mathrm{~cm}$ & STL-6303E \\
\hline $2005 / 10 / 10$ & 90 & 98 & 19852 & $80 \mathrm{~cm}$ & ST-7E \\
\hline $2005 / 10 / 11$ & 240 & 248 & 17872 & $50 \mathrm{~cm}$ & STL-6303E \\
\hline $2005 / 10 / 18$ & 90 & 98 & 16532 & $80 \mathrm{~cm}$ & ST-7E \\
\hline $2005 / 10 / 26$ & 90 & 98 & 20095 & $80 \mathrm{~cm}$ & ST-7E \\
\hline 2006/09/12 & 60 & 63 & 15919 & $80 \mathrm{~cm}$ & STL-1001E \\
\hline $2006 / 09 / 13$ & 60 & 63 & 21312 & $80 \mathrm{~cm}$ & STL-1001E \\
\hline $2006 / 09 / 20$ & 60 & 63 & 23056 & $80 \mathrm{~cm}$ & STL-1001E \\
\hline $2006 / 09 / 20$ & 240 & 247 & 17321 & $50 \mathrm{~cm}$ & STL-6303E \\
\hline $2006 / 09 / 21$ & 60 & 63 & 22489 & $80 \mathrm{~cm}$ & STL-1001E \\
\hline $2006 / 09 / 21$ & 180 & 187 & 21441 & $50 \mathrm{~cm}$ & STL-6303E \\
\hline $2006 / 09 / 22$ & 60 & 63 & 22299 & $80 \mathrm{~cm}$ & STL-1001E \\
\hline $2006 / 09 / 22$ & 180 & 187 & 1922 & $50 \mathrm{~cm}$ & STL-6303E \\
\hline $2006 / 09 / 22$ & 420 & 427 & 13388 & $50 \mathrm{~cm}$ & STL-6303E \\
\hline $2006 / 09 / 23$ & 90 & 93 & 22008 & $80 \mathrm{~cm}$ & STL-1001E \\
\hline $2006 / 09 / 23$ & 180 & 187 & 20595 & $50 \mathrm{~cm}$ & STL-6303E \\
\hline $2006 / 09 / 24$ & 60 & 63 & 9805 & $80 \mathrm{~cm}$ & STL-1001E \\
\hline $2006 / 09 / 24$ & 240 & 247 & 24678 & $50 \mathrm{~cm}$ & STL-6303E \\
\hline 2006/09/27 & 90 & 93 & 9470 & $80 \mathrm{~cm}$ & STL-1001E \\
\hline 2006/09/27 & 240 & 247 & 3205 & $50 \mathrm{~cm}$ & STL-6303E \\
\hline $2006 / 10 / 08$ & 240 & 247 & 11601 & $50 \mathrm{~cm}$ & STL-6303E \\
\hline $2006 / 10 / 09$ & 240 & 247 & 19808 & $50 \mathrm{~cm}$ & STL-6303E \\
\hline $2006 / 10 / 10$ & 240 & 247 & 19241 & $50 \mathrm{~cm}$ & STL-6303E \\
\hline 2006/10/11 & 240 & 247 & 19721 & $50 \mathrm{~cm}$ & STL-6303E \\
\hline 2006/10/12 & 60 & 63 & 18105 & $80 \mathrm{~cm}$ & STL-1001E \\
\hline $2006 / 10 / 16$ & 60 & 63 & 17602 & $80 \mathrm{~cm}$ & STL-1001E \\
\hline $2006 / 10 / 16$ & 240 & 247 & 16763 & $50 \mathrm{~cm}$ & STL-6303E \\
\hline 2006/10/17 & 60 & 63 & 13798 & $80 \mathrm{~cm}$ & STL-1001E \\
\hline 2006/10/17 & 120 & 123 & 4052 & $80 \mathrm{~cm}$ & STL-1001E \\
\hline 2006/10/17 & 240 & 247 & 18758 & $50 \mathrm{~cm}$ & STL-6303E \\
\hline $2006 / 10 / 26$ & 60 & 63 & 16031 & $80 \mathrm{~cm}$ & STL-1001E \\
\hline $2006 / 10 / 27$ & 60 & 63 & 19739 & $80 \mathrm{~cm}$ & STL-1001E \\
\hline $2006 / 10 / 30$ & 40 & 43 & 11609 & $80 \mathrm{~cm}$ & STL-1001E \\
\hline $2006 / 11 / 15$ & 240 & 247 & 18264 & $50 \mathrm{~cm}$ & STL-6303E \\
\hline
\end{tabular}

raising and falling edges would lie significantly below the sine fit flanks. Although the light curve solution presented by Nagel et al. (2006) still suffers from a degeneracy in mass and radius for the PG 1159 component, this light curve shape emphasizes the superiority of their more realistic model over a plain sine fit and validates their interpretation of the observed brightening as a reflection effect.

The refined ephemeris and period determination will allow a precise extrapolation of the ephemeris well into the next observing seasons, and facilitate the phasing of radial velocity measurements. This additional information will help to lift the existing degeneracy, so that we will be able to present a more elaborate light curve model in a future paper, and derive the masses in the system.
Acknowledgements. We thank Sylvia Brandert, Sebastian Wende, Jens Adamczak, Agnes Hoffmann, Ralf Kotulla, Simon Hügelmeyer, Markus Hundertmark, and Johannes Fleig for supporting the 2006 observations; and Klaus Reinsch for encouragement and technical support. We gratefully acknowledge the initialization of this project by Boris Gänsicke who first directed our attention to this special object.

\section{References}

Nagel, T., Schuh, S., Kusterer, D.-J., et al. 2006, A\&A , 448, L25 Schuh, S., Dreizler, S., Deetjen, J. L., \& Göhler, E. 2003, Balt. Astron., 12, 167

Werner, K. \& Herwig, F. 2006, PASP , 118, 183 